\title{
Anxiety as a barrier to risk management
}

\section{J Firth-Cozens}

Leaders need to recognise that anxiety is an inevitable part of caring for patients and that, without the building of trust, it may affect risk management by reducing the reporting of error.

$\mathrm{T}$ he paper by Kuhn and Youngberg ${ }^{1}$ in this issue of QSHC takes an historical approach to the evolution of risk management, following it from past failures into the path for its future success. The essence of the change will occur with the primary goal of risk management moving from the protection of the financial concerns of the organisation to the protection of its patients in terms of improved safety and quality of care. For decades risk management has sat uneasily beside quality systems, and it is only with the integration of these systems into one which has good patient care as its ultimate goal-clinical governance in the UK - that there is the possibility of success

Nevertheless, while the structural integration of quality systems may be relatively straightforward, the psychological integration of the concepts of "quality = good" and "error = bad" will take much longer to address. It is this splitting off of painful anxiety about real or potential errors-the fear, guilt and shame that it involves ${ }^{2}{ }^{3}$ - which lays in the uncomfortable unconscious of health care and which makes the task of risk management so difficult. Many years ago Isobel Menzies-Lyth conducted her seminal exploration of the ways in which healthcare organisations and professions structure themselves and their procedures in order to protect themselves from the anxiety associated with caring for distressed, diseased, and dying patients. ${ }^{4}$ Her work is embedded primarily in the provision of good care; however, accepting that error is not rare but a strong possibility for all individuals and that such error can lead to the very distress, disease, and death that we strive to relieve, cure or avoid may make the anxiety she explores potentially unbearable. Our new risk management depends upon the open discussion of error but, as we saw in Bristol, defences against anxiety are likely to create new barriers to seeing and accepting this error.

I would suggest that it is these barriers against anxiety which have until now stopped risk management being successfully integrated into the whole system of improving patient care: risk managers have traditionally been kept physically separate from other systems, seen as something of a joke, a burden clinicians sometimes have to bear, interested only in the tedious. These are the defences of a staff hard pressed, often underresourced and, not surprisingly, unwilling to get involved with the anxiety provoking idea that error is an ordinary everyday event.

\section{"It is the splitting off of painful anxiety about real or potential errors ... . which makes the task of risk management so difficult"}

The addressing of near misses is seen as an essential part of the new risk management ${ }^{5}$ and should make the discussion of error less difficult by focusing on potential rather than actual problems. However, there is also the argument that people adapt their behaviour more determinedly when a failure or disaster has happened to them rather than to someone else. For example, those in the town next to one hit by hurricane Hugo did little to protect their homes from future hurricanes, while those in the damaged town built strong defences for the future. ${ }^{67} \mathrm{~A}$ focus on near misses seems intuitively as useful in health care as it was in air travel, but we simply do not know the extent to which this will be offset by a lack of salience to overstretched staff. This, along with so many of the fundamental questions about patient safety, shows the need for a well funded and carefully considered programme of research for this crucial area.

The authors focus upon leadership as one way towards the new culture where error will be discussed and addressed within the whole system rather than by individual staff members. As they suggest, leaders need to attend not just to the financial business case but also to the moral one if they are to succeed in providing better patient safety. This moral case will include leaders taking on issues of trust-for example, that patients will be told the truth, that staff will be treated justly, and systems will be tackled to change what went wrong. Trust will be engendered through clarity about accountability, through honesty, and by consistency on the part of the leaders concerned. ${ }^{8}$ Finally, these leaders need to be aware of the anxiety faced by staff and by themselves in the provision of health care. It is so easy for a Chief Executive to avoid seeing what clinicians and patients have to face daily in their organisation, but it will not help trust to develop if they do.

Anxiety is thus a fundamental part of health care; not something we can change but something to be aware of so that the defences we build against it do not end up making the risk management of the future as unsuccessful as that of the past.

Qual Saf Health Care 2002;11:115

Correspondence to: Professor J Firth-Cozens, London Deanery, 20 Guilford Street, London WCIN IDZ, UK

jfirth-cozens@londondeanery.ac.uk

\section{REFERENCES}

1 Kuhn AM, Youngberg BJ. The need for risk management to evolve to assure a culture of safety. Qual Saf Health Care 2002:11:158-62.

2 Leape LL, Woods DD, Hatlie M, et al. Promoting patient safety by preventing medical error. JAMA 1998;280:1444.

3 Davidoff F. Shame: the elephant in the room. Qual Saf Health Care 2002;11:2-3.

4 Menzies-Lyth I. Containing anxiety in institutions. London: Free Associations Press, 1988

5 Department of Health. An organisation with a memory. Report of an Expert Group on Learning from Adverse Events in the NHS chaired by the Chief Medicial Officer. London: The Stationery Office, 2000.

6 Firth-Cozens J. Cultures for improving patient safety through learning: the role of teamwork. Qual Health Care 2001;10(suppl II):ii26-31.

7 Norris FH, Smith T, Kaniasty K. Revisiting the experience behavior hypothesis: the effects of hurricane Hugo on hazard preparedness and other self-protective acts. Basic Appl Soc Psychol 1999;21:37-47.

8 Firth-Cozens J, Mowbray D. Leadership and the quality of care. Qual Health Care 2001;10(suppl II):ii3-7. 
Risk management

\section{Risk management or safety first?}

\section{G E Knox}

\section{Is a culture of safety the necessary prerequisite for allowing risk management to evolve?}

$\mathrm{T}$ he historical summary of risk management by Kuhn and Youngberg ${ }^{1}$ in this issue of QSHC concludes with the provocative challenge: "Those risk managers who accept change and think of new ways to embed risk management principles into their organization to help create meaningful and sustainable change will prosper. Those who don't should get out now. They are destined to fail and to fail their organizations."

This judgment may be a bit premature, too focused on the individual risk manager, and actually reverse cause and effect-that is, rather than "a need for risk management to evolve to assure a culture of safety", perhaps the opposite is true. Is it possible that a culture of safety (high reliability) is the necessary prerequisite for allowing risk management to evolve? It is possible that, in a highly reliable safety culture, the risk management function as outlined by Kuhn and Youngberg may turn out to have minimal functional usefulness.

Svedung and Rasmussen ${ }^{2}$ have recently suggested that the following considerations are necessary for effective management of future risk:

(1) It is ... becoming increasingly difficult to explain accident causation by (retrospective) analysis of local factors within a work system. Safety and risk management increasingly become sociotechnical system problems (rather than the study of accidents themselves).

(2) A very aggressive and competitive environment tends to focus the incentives of decision makers on short term financial criteria rather than on long term criteria concerning welfare, safety and environmental impact . . . court reports from several recent accidents show that they have not been caused by coincidence of independent failures and human errors but by a systematic migration of organizational behavior toward accidents under the influence of pressure toward cost effectiveness in an aggressive competitive environment.
(3) Risk management has been reactive and primarily guided by studies of past accidents and incidents. It is suggested that future risk management strategies be based not on analysis of accidents, but rather on normal work practices and sociocultural factors that shape those work practices in an actual work place. It is therefore entirely possible that the ultimate "root cause" of any accident is production pressure, the origins of which lie outside the domain(s) currently considered manageable in the framework proposed by Youngberg and Kuhn.

This is not to suggest that risk transfer and claims resolution will cease to be important corporate functions. Healthcare accidents are, after all, accidents. While it may be possible to decrease the probability of an accident occurring, given the complexity of human nature and technology that are integral to the delivery of health care, mishaps will remain a natural part of our delivery system. Indeed, as Kuhn and Youngberg point out, the ability to "negotiate risk transfer agreements with steadily declining costs" contributes operating capital and increased margins, both of which have been shown to decrease mortality, thereby increasing patient safety and mitigating risk. ${ }^{3}$

Kuhn and Youngberg raise the interesting question: "How could we (risk managers) have worked so hard and accomplished so little?" This question in turn creates others. How can anyone know what has not been accomplished? How is it possible to measure accidents or injury that have been prevented and therefore did not occur? It is impossible to know what the total cost of risk would have been had an attempt to manage healthcare risk (even with paradigms suggested as not effective) not been undertaken

When pricing and rates are soft, the very hard work of negotiating favorable terms ironically ensures that incentives do not exist to thwart what the German sociologist Ulrich Beck has described as "an acceptance of uncertainty and organized irresponsibility". ${ }^{4}$ By contrast, the prediction of dramatic environmental change such as the twin tower disaster in New York on 11 September 2001 that leads to an industry wide knee jerk reaction is no more the purview of individual risk managers than of corporate senior management itself.

Indeed, some responsibility for whatever proposed shortcomings are inherent in the current model of healthcare risk management must include corporate governance and accountability by senior management. As Kuhn and Youngberg point out, managing risk is about corporate design and improvement and changing systems of work rather than simply a staff function assigned to an office or someone labelled "risk management". Integrating the work of risk into organizational and managerial culture and making it an explicit step in the decision making process is critical to future successful management of corporate healthcare risk.

Managing future risk is not, as suggested by Kuhn and Youngberg, about "embedding risk management principles into healthcare organizations"- that is, reporting for the purpose of analyzing, characterizing and trending accidents, incidents and near misses. Rather, it is about replacing current risk management principles with those found in organizational development, the social sciences, financial modelling, knowledge management, and story telling. The chaotic and complex nature of healthcare risk suggests that anything else will fall short.

Qual Saf Health Care 2002;11:116

Correspondence to: Dr G E Knox, Director, Patient Safety, Children's Hospitals and Clinics, 2525 Chicago Avenue, Minneapolis, MN 55404, USA; eric.knox@childrenshc.org

\section{REFERENCES}

1 Kuhn AM, Youngberg BJ. The need for risk management to evolve to assure a culture of safety. Qual Saf Health Care 2002;11:158-62.

2 Svedung I, Rasmussen J. Graphic representation of accident scenarios: mapping system structure and the causation of accidents. Safety Sci 2002;40:397-417.

3 Shortell SM, Rousseau DM, Giles RR, et al. A national program to improve the quality of ICU services. Final report submitted to the Health Care Financing Administration, April 1991.

4 Beck U. Risk society: towards a new modernity. London: Sage, 1992. 


\section{Patient centred care: essential but probably not sufficient}

\section{K W Kizer}

One of the many lessons to emerge from the analysis of the care of children in the cardiac unit at the Bristol Royal Infirmary is the importance of engaging patients in decisions about their health and health care. This is a message that has relevance to all healthcare professionals in all clinical settings. Patient centredness is crucial for good quality care, but achieving genuine patient centred care throughout health services will require transformation of systems as well as attitudes. In this issue (pp 186-8) we have reproduced Angela Coulter's paper "After Bristol: putting patients at the centre", first published in the BM in March 2002.

$\mathrm{T}$ he determinants of health and illness are not just biological, nor is a person's response to injury or illness. Patients live in families and communities of various types. They often work in less than healthy or even in hazardous occupations, and they relax in activities that may be health promoting or not. Patients live within a larger political, cultural, and environmental context that further affects both them and their families or communities. All of these "less biological" aspects of a patient's life influence his or her state of health or condition of illness. Indeed, health and illness are integrated phenomena-that is, they integrate biological, social and cultural, economic, political, occupational and environmental, recreational and other aspects of an individual's life. The specific health effect of these disparate influences is complex and often is much more important than traditional health care has factored into customary approaches to diagnosis and treatment. As Coulter so clearly espouses in her prescription for patient centred care, ${ }^{1}$ cognizance of these patient related factors is crucial for effective treatment.

Coulter's prescription for redesigning health services is sound and timely, as far as it goes. The need for putting patients at the centre of the healthcare universe may have been especially highlighted by the unfortunate events at the Bristol Royal Infirmary, ${ }^{2}$ but the need to redesign the healthcare delivery system to be more responsive to the perspectives and needs of patients is evident every day in clinics, hospitals, nursing homes, and other care facilities throughout the delivery system. Restructuring healthcare delivery to be patient centred will require a fundamental transformation of healthcare operations, which will necessitate a sustained and concerted effort and which will be accompanied by a certain amount of pain. If successfully executed, however, the end result will be more effective use of time and resources, reduced costs, improved coordination and continuity of care, and better outcomes. If done correctly, the transformation to a patient centred healthcare delivery system will substantially improve the quality of care, as viewed by both patients and caregivers, and it will almost certainly decrease the per patient cost of care.

A key element in redesigning the healthcare system to be more patient centred will be preserving and, in many cases, enhancing the caregiver-patient relationship. This intimate relationship is the medium by which information, feelings, fears, concerns, and hopes are exchanged between caregiver and patient. The integrity of this relationship is foundational for successful diagnosis and treatment. It is also a key determinant of how satisfying is the care experience for both patient and caregiver. Indeed, the process of interaction between caregiver and patient is often the most therapeutic aspect of the healthcare encounter.

While recognising the essentiality of transforming health care to be more patient centred for all the reasons that were articulated by Coulter, we also must be mindful that, just as health is an integrative phenomenon, so is health care. Indeed, health care involves complex caregivers and with the ambient environment that significantly impacts on therapeutic outcomes.

Modern health care is the most complex activity ever undertaken by relationships between and among human beings. It involves highly complicated technology that can seriously harm as well as miraculously heal. It is a team activity with more than $80 \%$ of the hands-on care being provided by nonphysicians. The myriad of specialised caregivers are often focused on only one aspect of the patient's care, so there are many care "hand offs" among them. Information about the technical aspects of care must be communicated and acted upon by the various caregivers in a coordinated manner. These complex and multifaceted interactions need to be orchestrated in consistent and predictable ways that are mutually satisfying to both patient and caregiver. This is a social process that is subject to the cultural, economic, and political dimensions inherent to the care processes.

Just as the determinants of health and illness are not just biological, the determinants of health care are neither just biological nor just technical. Health care is an integrated phenomenon that has its own social, cultural, economic, and political dimensions that are often as important, or more so, than the technical dimensions on which we more often focus. Effective health care must integrate the biological, technical, social, cultural, economic, political, and other aspects of both patients and caregivers. These dimensions of care have to be integrated into the systems of care. We need to view health care as being provided by "treatment families", "treatment teams", or "therapeutic communities", as opposed to something done by individual caregivers. Just as with patient's families and communities, health care families and communities may operate with varying degrees of functionality and be more or less effective.

Unfortunately, the dynamics of the relationships that caregivers have with each other and with the larger therapeutic communities in which they practice have not yet been well studied and are only rarely addressed in healthcare teaching. To improve the likelihood that the various therapeutic entities will promote health care, caregivers need to be trained in techniques of team based problem solving and team based care management. These are areas that have only recently begun to be addressed in health care, with nursing and anesthesia being the most progressive so far. ${ }^{3-5}$ Models of such include aviation style crew resource management, MedTeams, and anesthesia crisis management. Health care must also address the cultural and political contexts of care itself and how these interface with the larger societal, cultural and political contexts, each of which may facilitate or impede optimal therapeutic outcomes. Again, only recently have we begun to rigorously analyse and experiment with these dimensions of the care process. ${ }^{67}$ 
The patient centred model of care is essential because it promotes a "whole person" approach to care that recognizes the larger context in which patients live and function. However, it alone is not likely to be sufficient because it does not explicitly embrace the interdisciplinary and sociocultural nature of health care itself. The integrative nature of health care will have to be addressed if health care is truly to operationalize the patient centred model of care and if we are to achieve the improvement in healthcare quality that is so much needed.

Qual Saf Health Care 2002;11:117-118
Correspondence to: K W Kizer, President and Chief Executive Officer, The National Quality Forum, Washington, DC, USA;

KWKizer@cs.com

\section{REFERENCES}

1 Coulter A. After Bristol: putting patients at the center BM 2002:324:648-51.

2 Bristol Royal Infirmary Inquiry. Learning from Bristol: the report of the public inquiry into children's heart surgery at the Bristol Royal Infirmary 1984-1995. London: Stationery Office, 2001; http:// www.bristol-inquiry.org.uk (accessed 5 February 20011)

3 Byrne AJ, Sellen AJ, Jones JG, et al. Effects of videotape feedback on anaesthetists' performance while managing simulated anesthetic crises: a multicentre study. Anaesthesia 2002;57: 169-82.

4 Zinober JW. Building a sense of teamwork within the organization. Getting along with your practice partners. Med Group Manage J 1991;38:36-9

5 Hetherington LT. Becoming involved: the nurse leader's role in encouraging teamwork. Nurs Admin Q 1998;23:29-40.

6 Rafferty AM, Ball J, Aiken LH. Are teamwork and professional autonomy compatible, and do they result in improved hospital care? Qual Health Care $2001 ; 10: 32-7$.

7 Ratto M, Propper C, Burgess S. Using financial incentives to promote teamwork in health care. J Health Serv Res Policy 2002;2:69-70.

\section{Impact of national guidelines on use of surgery for glue ear in England}

\section{Keeley}

\section{Change in practice follows Effective Health Care bulletin-post hoc or propter hoc?}

A significant proportion of medical activity is based on custom and practice rather than on sound evidence of effectiveness. It is a matter of concern worldwide to understand how best to encourage doctors to stop doing things that don't work. The paper by Black and Hutchings ${ }^{1}$ in this issue of QSHC offers a fascinating account of the time trends in rates of surgery for glue ear and the possible impact of the 1992 Effective Health Care bulletin which outlined the reasons to doubt the effectiveness of this common surgical procedure. $^{2}$

Rates of glue ear surgery in NHS hospitals, which were already falling slowly before the bulletin appeared, fell from 120 per 10000 in $1992 / 3$ to 68 per 10000 in $1997 / 8$. The fall in intervention rate remains significant-though less dramatic —when private hospital activity is taken into account. Several contextual factors are identified to explain why the message of the Effective Health Care bulletin did not fall on deaf ears: a downward trend had already begun as both otolaryngologists and GPs were beginning to doubt the value of grommets, media reports of these doubts were influencing parents' readiness to request referral, and the purchaser/provider split encouraged closer scrutiny of the effectiveness of interventions. A further contextual factor not brought out by the authors is the relative freedom of the British healthcare system from fee for service incentives for medical activity of dubious worth.

Experience from another study suggests that the rate of referral by general practitioners for glue ear fell by $50 \%$ during this period, but practitioners in both primary care and hospital interviewed in a separate qualitative study reported little awareness of or influence by the Effective Health Care bulletin. ${ }^{3}$ We cannot always easily identify what makes us change what we do, and when we do change our practice we are sometimes reluctant to acknowledge, even to ourselves, that we have done so. Many of opinion, but in this case the Effective Health Care bulletin looks to have made a real impact—or at least to have benefited

\section{To send a rapid response}

Visit the website (www.qualityhealthcare.com), open the paper of interest, click on "Submit a response" and follow the instructions on screen. different factors contribute to a climate from an extraordinary serendipity of timing.

The ancient Greeks had a wordkairos-for doing things at the right time. The Effective Health Care bulletin was pushing at an opening door. But in a highly debatable conclusion the authors of this study suggest that producers of guidelines should "focus on those topics for which the environment is likely to be conducive to change" and "avoid expending effort in areas where change is unlikely". We should remember that powerful forces are at work to shape the environment in which clinical evidence is presented-including commercial interests and episodes of spectacular unwisdom from factions in politics and the media such as we are currently witnessing over MMR immunisation. Guideline production, in common with other forms of scientific activity, should not confine itself to picking winners. What do you think? Whether you are a writer or a reader, or just a weary recipient of guidelines, the journal would like to hear your views.

Qual Saf Health Care 2002;11:118

Correspondence to: Dr D Keeley, General Practitioner, Thame, Oxon OX9 3JZ; yc23@dial.pipex.com

\section{REFERENCES}

1 Black N, Hutchings A. Reduction in the use of surgery for glue ear: did national guidelines have an impact? Qual Saf Health Care 2002;11:121-4.

2 NHS Centre for Reviews and Dissemination. The treatment of persistent glue ear in children. Effective Health Care 1992;1(4): 1-16.

3 Dopson S, Miller R, Dawson S, et al. Influences on clinical practice: the case of glue ear. Qual Health Care 1999;8:108-18. 


\section{Simulation for training is effective when ...}

\section{E Salas, C S Burke}

Simulation can benefit the medical community by training both individuals and teams to reduce human error and promote patient safety.

\section{SIMULATION FOR TRAINING IS EFFECTIVE WHEN . . .}

There is no question that simulation can be an effective tool for training complex skills. There is some evidence that it works. ${ }^{1}$ But it is only a tool. As with any tool, in order to be effective it must be used appropriately. We commend the paper by Satish and Streufert ${ }^{2}$ in this issue of QSHC for highlighting the role that simulation may play in both training and assessment within the medical community, as well as the recognition that effective simulation must: (1) be built on underlying theory (they use complexity theory), (2) use structured exercises, and (3) assess performance and provide feedback. However, some additional observations about simulation are warranted so that scientists and training developers within the medical community do not fall into some common myths and misconceptions known to exist regarding training in general, as well as the use of simulation for training. ${ }^{3}$ We therefore present a few observations based on the science of training ${ }^{14}$ and our experience in aviation and military environments about when simulation is effective for training. ${ }^{56}$ Simulation for training is effective when ...

\section{(1) ... instructional features are} embedded within the simulation Simulations to facilitate learning need to be designed around key instructional components-that is, simulation based training must have a series of links that create a learning environment (fig 1). One instructional strategy that has been successfully used in aviation and military environments and embeds the above instructional features is the event based approach to training (EBAT). This strategy relies on the "a priori" embedding of multiple events into the scenario at different time intervals. These events serve as cues for trainees to exhibit competencies targeted in training. ${ }^{7}$ In turn, these cues serve as measurement and feedback opportunities. Advantages to this approach include:

- ensuring opportunities to exhibit targeted behavior are presented;

- scenario control while giving the appearance of a free flowing scenario;

- increasing the ease with which competencies can be measured;

- providing standardization across trainees.

Simulation can therefore only create opportunities for learning if instructional features are built into it.

\section{(2) ... carefully crafted scenarios} are embedded within the simulation Satish and Streufert ${ }^{2}$ suggest that the SMS simulation can define scenarios "a priori". However, further clarification is needed regarding the factors that drive scenario parameters. One must remember that, in simulation based training,

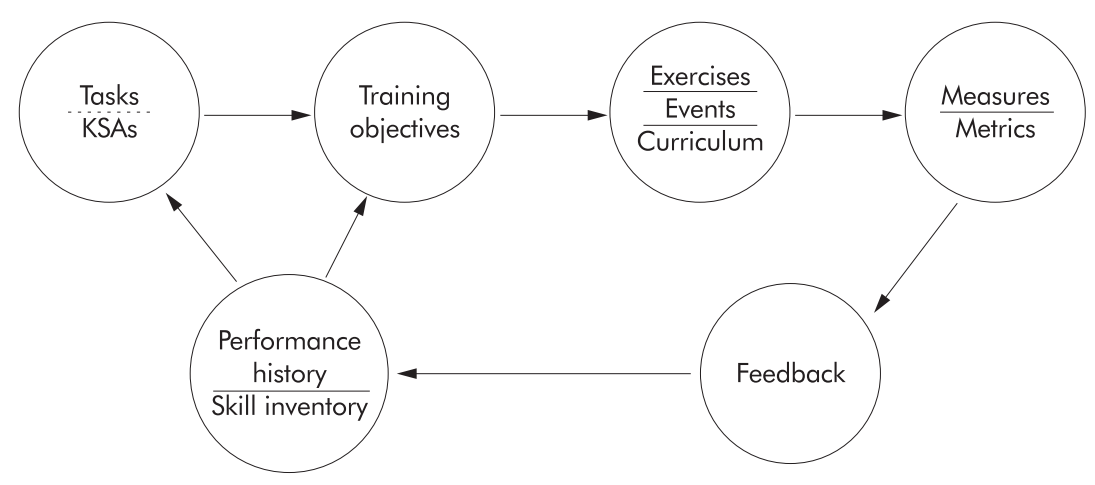

Figure 1 Components of scenario based training (adapted from Cannon-Bowers et a ${ }^{16}$ ). the scenario(s) are the curriculum so they must be carefully storyboarded. This could be facilitated by performing a cognitive task analysis (CTA). A CTA should help in determining the content of the scenarios since it will uncover the cues expected to be used to perform complex tasks. In addition, scenarios should build events into scripts. These inserted events serve as "triggers" and provide known opportunities to both practise and assess important behaviors. Scenarios are therefore a key component for simulation to facilitate learning and cannot be left to chance or created without a learning outcome in mind.

\section{(3) ... the simulation contains opportunities for assessing and diagnosing individual or team} performance

As noted above, we agree with Satish and Streufert $^{2}$ that simulation based training will only be effective to the extent that trainee competence can be assessed. There are two points to this statement.

Firstly, simulation based training must provide measurement opportunities that ease the burden on those responsible for measurement. More specifically, simulations that use pre-scripted learner focused scenario events not only ensure that relevant competencies are being assessed, but ease the assessment process as instructors know when key events will occur.

Secondly, not only does simulation need to build in opportunities for the assessment of performance, but also these measurement opportunities must provide the basis for diagnosing skill deficiencies. In other words, it is not enough that the simulation provides opportunities to capture performance outcomes, but it must also (as much as possible) capture the moment-tomoment actions and behaviors. These process oriented measurements are much richer for training purposes; they are also the most difficult to capture and may require human intervention. Simulations that include measurement systems which only capture outcome measures (such as quality or quantity) do not allow those responsible for training to diagnose performance; they do not offer information on how to improve performance. Performance measurement is paramount to training and, without it, simulations are just that-simulations.

\section{(4) ... the learning experience is guided}

We have all heard the saying "practice makes perfect". Similarly, it has been argued that experience-that is, practice-can make an excellent teacher because it generates knowledge within a meaningful context. ${ }^{2}$ The conditional nature of both these statements needs to 
be highlighted-that is, practice or experience in and of itself does not equal learning. Trainees who are given unguided practice often:

- learn the wrong thing;

- do not focus on practising the right behaviors;

- may spend too much time on only one particular aspect of training;

- may not be able to transfer the skills to the job.

To maximize the learning experience, practice must be guided (through carefully crafted scenarios and diagnostic timely feedback) so that trainees remain focused on learning key competencies.

(5) ... simulation fidelity is matched to training requirements

When using simulations for training purposes it is often assumed that more is better; this is not true. For example, research has found that use of high physical fidelity simulations in training did not transfer or had very little effect on actual job tasks. ${ }^{8}$ Similarly, research has successfully used low fidelity PC based simulations to train complex individual and teamwork skills. ${ }^{9-12}$ The level of simulation fidelity needed should be driven by the cognitive and behavioral requirements of the task and the level needed to support learning.'

Finally, simulation for training is effective when ..

\section{(6) ... there is a reciprocal partnership between subject matter experts and learning/training specialists}

Learning is a behavioral/cognitive event. Training is about imparting longlasting change in trainees. It is about creating a context where key competitiveness can be practised, assessed, diagnosed, remedied, and reinforced. To do that requires a partnership between task experts and those who know about the design and delivery of training. No one can do it effectively alone. Both parties have something to contribute: subject matter experts articulate task requirements and needs while training specialists create learning environments. Both are needed and the medical community should foster it

\section{CONCLUSION}

Simulation is an effective tool for training complex skills. The military and aviation environments have invested heavily in simulation based training and, although further multilevel assessments need to be conducted, initial data regarding its effectiveness are encouraging. ${ }^{13-1}$ However, simulation is only a tool, and training developers and practitioners must rely on the science of training to maximize the effectiveness of it. There are known principles. Our recommendation is to apply them, and to develop a partnership with those who understand what it takes to design and deliver effective training.

Simulations must be designed so that: (1) instructional features are embedded within the simulation, (2) carefully crafted scenarios are embedded that contain opportunities for performance measurement and diagnostic feedback, (3) the learning experience is guided, and (4) simulation fidelity is matched to task requirements. Keeping all this in mind, the medical community can gain great benefits from using simulation to train both individuals and teams to reduce human error and promote patient safety.

Qual Saf Health Care 2002;11:119-120

Authors' affiliations

E Salas, C S Burke, Institute for Simulation \& Training and Department of Psychology, University of Central Florida, Orlando, FL 32826, USA

Correspondence to: Dr E Salas, Institute for Simulation \& Training, University of Central Florida, 3280 Progress Drive, Orlando, FL 32826,USA; esalas@pegasus.cc.ucf.edu

\section{REFERENCES}

1 Tannenbaum SI, Yukl G. Training and development in work organizations. Ann Rev Psychol 1992;43:399-441.
2 Satish U, Streufert S. Value of a cognitive simulation in medicine: towards optimizing decision making performance of healthcare personnel. Qual Saf Health Care 2002;11:163-7.

3 Salas E, Cannon-Bowers JA, Rhodenizer L, et al. Training in organizations: myths, misconceptions, and mistaken assumptions. Personnel Human Resources Manage 1999:17:123-61.

4 Salas E, Cannon-Bowers JA. The science of training: a decade of progress. Ann Rev Psychol 2001:52:471-99.

5 Salas $\mathbf{E}$, Bowers CA, Rhodenizer L. It is not how much you have but how you use it: Toward a rational use of simulation to support aviation training. Int J Aviat Psychol 1998:8:197-208.

6 Cannon-Bowers JA, Salas E, eds. Decision making under stress: implications for training and simulation. Washington, DC: American Psychological Association, 1998.

7 Fowlkes J, Dwyer D, Oser R, et al. Event-based approach to training. Int J Aviat Psychol 1998;8:209-22.

8 Taylor HL, Lintern G, Koonce JM, et al. Scene content, field of view and amount of training in first officer training. In: Jensen RS, ed. Proceedings of the 7th International Symposium on Aviation Psychology. Columbus: Ohio State University, 1993: 753-7.

9 Gopher D, Weil M, Bareket T. Transfer of skill from a computer game trainer to flight. Human Factors 1994;36:387-405.

10 Jentsch F, Bowers CA. Evidence for the validity of PC-based simulations in studying aircrew coordination. Int J Aviat Psychol 1998;8:243-60

11 Prince C, Jentsch F. Aviation crew resource management training with low-fidelity devices. Improving teamwork in organizations: applications of resource management training. Mahwah, NJ: Lawrence Erlbaum Associates, 2001: 147-64

12 Taylor HL, Lintern G, Hulin CL, et al. Transfer of training effectiveness of a personal computer aviation training device. Int J Aviat Psychol 1999;9:319-35.

13 Salas E, Burke CS, Bowers CA, et al. Team training in the skies: does crew resource management (CRM) training work? Human Factors 2002 (in press)

14 Holzman RS, Cooper JB, Gaba D, et al. Anesthesia crisis resource management: real life simulation training in operating room crises. J Clin Anesth 1995;7:675-87.

15 Barach P, Satish U, Steuffert S. Heealthcare assessment and performance. Simulation Gaming 2001;32:147-51.

16 Cannon-Bowers JA, Burns JJ, Salas E, et al. Advanced technology for scenario-based training. In: Cannon-Bowers JA, Salas E, eds. Making decisions under stress: implications for individual and team training. Washington, DC: American Psychological Association, 1998: 365-74. 